\title{
Poincare Indices for Analyzing Meditative Heart Rate Signals
}

\author{
Atefeh Goshvarpour, Ateke Goshvarpour
}

Background: Poincare plots are commonly used to study the nonlinear behavior of physiologic signals. The aim of this study is to evaluate the Poincare plot indices of human heart rate signals during meditation.

Methods: $\quad$ For this purpose, heart rate time series of eight Chi meditators available in Physionet database were used. Poincare plots with lags of 1 and 6 were constructed, and the ratio of the minor axis to major axis (SD1/SD2) and the area of Poincare plots were calculated for each lag.

Results: The results show that the SD1/SD2 ratio increased significantly during meditation compared to that before meditation, especially the index measured from Poincare plots reconstructed with a lag of $6(p<0.05)$. In addition, in both lags, the area of Poincare plots decreased significantly during meditation compared to before meditation $(p<0.05)$.

\section{At a Glance Commentary}

Scientific background of the subject

Poincare plots are commonly used to study the non-linear behavior of physiologic signals. The aim of this study is to evaluate Poincare plot indices of human heart rate signals during meditation.

\section{What this study adds to the field}

According to our study, the comparative dynamic measures of the Poincare plot indices during and before meditation give more insight of the heart rate signals in a specific psychophysiological state.

Conclusion: The comparative dynamic measures of the Poincare plot indices during and before meditation give more insight of the heart rate signals in a specific psychophysiological state.

(Biomed J 2015;38:229-234)

Key words: heart rate signals, meditation, nonlinear dynamics, poincare plots

$\mathrm{T}^{\mathrm{t}}$ The analysis of heart rate variability (HRV) provides valuable information to assess the autonomous nervous system (ANS). Since information embedded in the HRV was found to be directly linked to health ${ }^{[1]}$ and cardiac diseases like heart failure, ${ }^{[2]}$ angina pectoris, ${ }^{[3]}$ and myocardial infarction, ${ }^{[4]}$ analysis of these signals has gained the favor of many researchers for a long time. In addition, its low-cost, non-invasive nature and effectiveness encourages the development of new HRV analysis methods to broaden and improve its applications.

Traditional techniques in the analysis of HRV are based on linear methods in time, frequency, or time-frequency domain. These methods have been extensively used to reveal the fundamental control of sympathetic and parasympathetic activities of ANS. ${ }^{[5]}$ Many researchers have concluded that nonlinear features are important in the study of HRV ${ }^{[6]}$ The analysis of time series in the nonlinear domain includes estimation of dynamical invariants from the reconstructed attractor. Poincare plot is one of these techniques. ${ }^{[7,8]}$

The Poincare plot is achieved from the representation of a time series into a Cartesian plane (or phase space), where the values of each pair of sequential elements of the time series define a point in the plot. The Poincare plot is a very simplified phase space with a dimension of two and delay or lag of one beat (i.e. each RR interval is plotted as a function of the previous RR interval). The Poincare plot gives a powerful visual contact with the RR time series by representing both short- and long-term variations included in the recording. ${ }^{[9]}$ The following indices are introduced to quantify the Poincare plot geometry: SD1, SD2, and the SD1/SD2 ratio ${ }^{[9,10]}$ Furthermore, the analysis of the Poincare plot is considered by some authors as based on nonlinear dynamics. ${ }^{[7,8,11]}$

From the Computational Neuroscience Laboratory, Department of Biomedical Engineering, Faculty of Electrical Engineering,

Sahand University of Technology, Tabriz, Iran

Received: Oct. 8, 2013; Accepted: Sep. 9, 2014

Correspondence to: Dr. Ateke Goshvarpour, Computational Neuroscience Laboratory, Department of Biomedical Engineering, Faculty of Electrical Engineering, Sahand University of Technology, Tabriz, Iran. New Sahand Town, Tabriz, Iran. Tel: 98-51-37625993;

Fax: 98-51-37625993; E-mail: ak_goshvarpour@sut.ac.ir

DOI: $10.4103 / 2319-4170.143528$ 
In a previous study, ${ }^{[11]}$ the width of the Poincare plot with different lags was evaluated on heart rate (HR) signals during meditation.

Meditation provokes physical and mental relaxation and enhances psychological balance and emotional stability. ${ }^{[12]}$ Moreover, it is acclaimed as a non-medicated treatment for some psychological disorders without any side effects, unlike medication. ${ }^{[13]}$

Many types of meditation techniques have been introduced. The one adopted here is Chinese Chi meditation. In this technique, the meditators sit quietly and breathe spontaneously while visualizing the opening and closing of a perfect lotus in the stomach.

On applying the statistical and spectral measures of HRV, it was found that meditation may have different effects on health, depending on the resonant peaks' frequencies. ${ }^{[14]}$ It has been shown that during meditation, the resonant peak occurs in the very low frequency (VLF), low frequency (LF), or high frequency (HF) range. Therefore, it may reset baroreflex sensitivity and enhance parasympathetic tone. ${ }^{[14]}$ Recently, evaluation of the higher-order spectra of HR patterns has shown that not only there are significant differences in them between rest and meditation, but also they are influenced by different types of meditation. ${ }^{[15]}$ Muralikrishnan et al. ${ }^{[16]}$ investigated the effects of Isha Yoga on cardiac ANS activity. They found that the Isha Yoga practitioners had a well-balanced activity of vagal efferents, an enhanced HRV, and sympathovagal balance, compared to non-practitioners. Litscher et al. ${ }^{[17]}$ compared HR and HRV responses to Tai Chi and jogging. Although the movements are slower in the Tai Chi training compared to jogging, similar maximum HRs were observed in both activities.

The present study was aimed to assess some other indices of the Poincare plot, with different lags, on HR signals during meditation. For this purpose, HR signals of two groups of subjects (before meditation and during meditation) were analyzed. Poincare plots with lags of 1 and 6 were constructed and the SD1/SD2 ratio and the area of Poincare plots were calculated for each lag. Finally, we present the results of the analysis of these features on data set and we conclude the study.

\section{METHODS}

\section{Data selection}

In the current study, HR signals from Physionet database ${ }^{[1]}$ were used prior to and during meditation. Subjects were at an advanced level of meditation training. All Chi meditators ${ }^{[1]}$ were graduate and post-doctoral students. In addition, they were relative novices in Chi meditation, most of them having begun their practice in meditation about 1-3 months before the data collection. The subjects were in good general health and did not follow any specific exercise routines.
The eight Chi meditators, five women and three men (age range 26-35, mean 29 years), wore a Holter recorder for about $10 \mathrm{~h}$ and did their ordinary daily activities. Roughly $5 \mathrm{~h}$ in the recording, each subject practiced $1 \mathrm{~h}$ of meditation. Meditation's beginning and ending times were delineated with event marks.

During these sessions, the Chi meditators were asked to sit quietly and listen to the taped guidance of the Master. The meditators were instructed to breathe spontaneously while visualizing the opening and closing of a perfect lotus in their stomach. The meditation session lasted about $1 \mathrm{~h}$. The sampling rate was $360 \mathrm{~Hz}$. Analysis was performed offline and meditations' beginning and ending times were determined with event marks.

\section{Time and frequency measures}

To gain more insight into the ANS activity during meditation, the spectral parameters of HRV signals were extracted. The measure in time domain comprising the mean HR was calculated. The spectral HRV parameters before and during meditation were calculated by applying Fast Fourier Transform (FFT).

According to the Task Force, ${ }^{[18]}$ for short time series the power spectrum can be categorized into three ranges of frequency as follows: (1) Power in the VLF range, $0.003-0.04 \mathrm{~Hz}$; (2) power in the LF range, $0.04-0.15 \mathrm{~Hz}$, and (3) power in the HF range, $0.15-0.4 \mathrm{~Hz}$.

\section{Poincare plots}

Poincare plot is a geometrical demonstration of a time series in a Cartesian plane. By plotting consecutive points of RR time series on phase space or Cartesian plane, a two-dimensional plot is constructed. ${ }^{[19]}$

Figure 1 demonstrates a standard Poincare plot of RR interval. ${ }^{[11]}$ Two basic descriptors of the plot are SD1 and

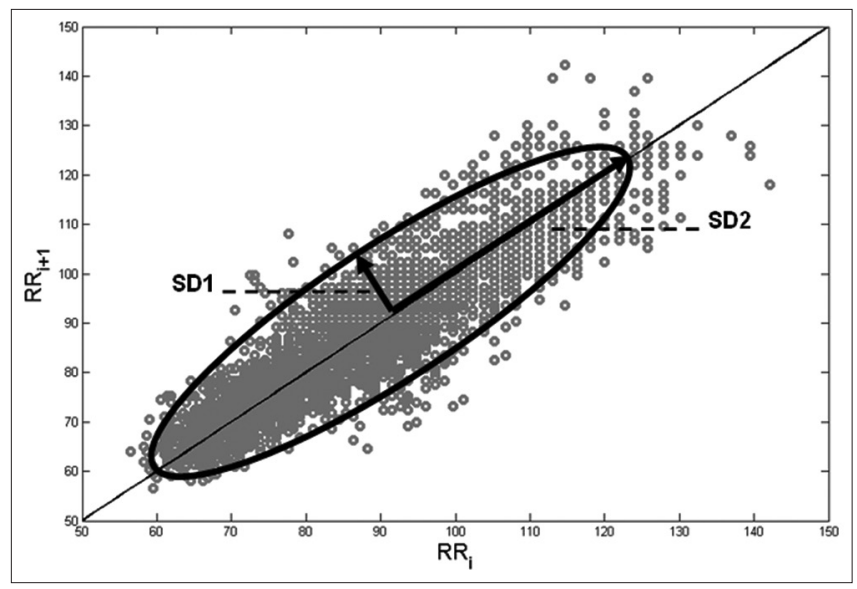

Figure 1: Standard Poincare plot. A standard Poincare plot (lag-1) of RR intervals before meditation (record C3). SD1 and SD2 represent the dispersion along minor and major axes of the fitted ellipse. 
SD2. The line of identity is the 45 imaginary diagonal line on the Poincare plot and the points falling on the imaginary line have the property $R R_{n}=R R_{n+1}$. The spreading of points perpendicular to the line of identity is represented by SD1, while the spreading along the line of identity is reflected by SD2. Fundamentally, SD1 and SD2 of Poincare plot are directly associated with the basic statistical measures, standard deviation of RR interval (SDRR) and standard deviation of the successive difference of RR interval (SDSD), which are given by the relations presented in equations (1) and (2).

$$
\begin{aligned}
\mathrm{SD}_{1}^{2}= & \frac{1}{2} \mathrm{SDSD}^{2}=\gamma_{\mathrm{RR}}(0)-\gamma_{\mathrm{RR}} \\
\mathrm{SD}_{2}^{2}= & 2 \mathrm{SDRR}^{2}-\frac{1}{2} \mathrm{SDSD}^{2}=\gamma_{\mathrm{RR}}(0) \\
& +\gamma_{\mathrm{RR}}(1)-2 \overline{\mathrm{RR}}^{2}
\end{aligned}
$$

Where $\gamma_{R R}(0)$ and $\gamma_{R R}(1)$ stand for the autocorrelation function for lag- 0 and lag- 1 of RR interval and the mean of $\mathrm{RR}$ intervals is shown by $\overline{\mathrm{RR}}$.

From equations (1) and (2), it is clear that the measures SD1 and SD2 are actually derived from the correlation and mean of the RR intervals time series with lag-0 and lag-1. The above equation sets are presented in unit time delay Poincare plot. Many researchers are interested in studying plots with different time delays to get a better insight in the time-series signal. Typically, the time delay is a multiple of the cycle length or the sampling time of the signal. ${ }^{[20]}$ By the choice of time delay, the dependency among the variables is controlled, and the most conventional analysis is performed with higher order linear correlation between points.

Plotting the 2D phase space with lag-m, the equations for the SD1 and SD2 can be written as:

$$
\begin{aligned}
& \mathrm{SD}_{1}^{2}=\gamma_{\mathrm{RR}}(0)-\gamma_{\mathrm{RR}}(\mathrm{m}) \\
& \Rightarrow \mathrm{SD}_{1}=\mathrm{F}\left(\gamma_{\mathrm{RR}}(0), \gamma_{\mathrm{RR}}(\mathrm{m})\right) \\
& \mathrm{SD}_{2}^{2}=\gamma_{\mathrm{RR}}(0)+\gamma_{\mathrm{RR}}(\mathrm{m})-2 \overline{\mathrm{RR}}^{2} \\
& \Rightarrow \mathrm{SD} 2=\mathrm{F}\left(\gamma_{\mathrm{RR}}(0), \gamma_{\mathrm{RR}}(\mathrm{m})\right)
\end{aligned}
$$

Where $\gamma_{R R}(m)$ is the autocorrelation function for lag-m RR interval. This suggests that the standard descriptors for any arbitrary $m$ lag Poincare plot is a function of the autocorrelation of the signal at lag- 0 and lag-m.

The SD1 is an index of instantaneous recording of the beat-to-beat variability and demonstrates the parasympathetic activity, while the SD2 shows HRV in long-term records and reflects the overall variability. SD1 and SD2 are combined to form two additional descriptors of Poincare plots: The ratio of SD1/SD2 and the area of Poincare plot. The former (SD1/SD2) shows the ratio between short and long variations of RR intervals $\mathrm{s}^{[10,21]}$ and the latter is defined by equation (4):

$$
S=\pi \times \mathrm{SD}_{1} \times \mathrm{SD}_{2}
$$

The area of the imaginary ellipse on the Poincare plot is shown by $S$.

\section{RESULTS}

Mean HR signals were calculated for eight subjects before and during meditation. Figure 2 depicts the variation of this parameter in two states. Applying the analysis of variance (ANOVA) test, mean HRV was significantly different before and during meditation $(p<0.05)$. As shown in Figure 3, power spectrum analysis revealed distinctive variations in the maximum power of before and during meditation $(p<0.05)$.

After Poincare plots were constructed, SD1 and SD2 values were calculated for each lag. During meditation, the value of the SD1 of Poincare plot with a lag of 1 was $2.2 \pm 0.6$ and with a lag of 6 was $7.3 \pm 1.7$. Unlike SD1

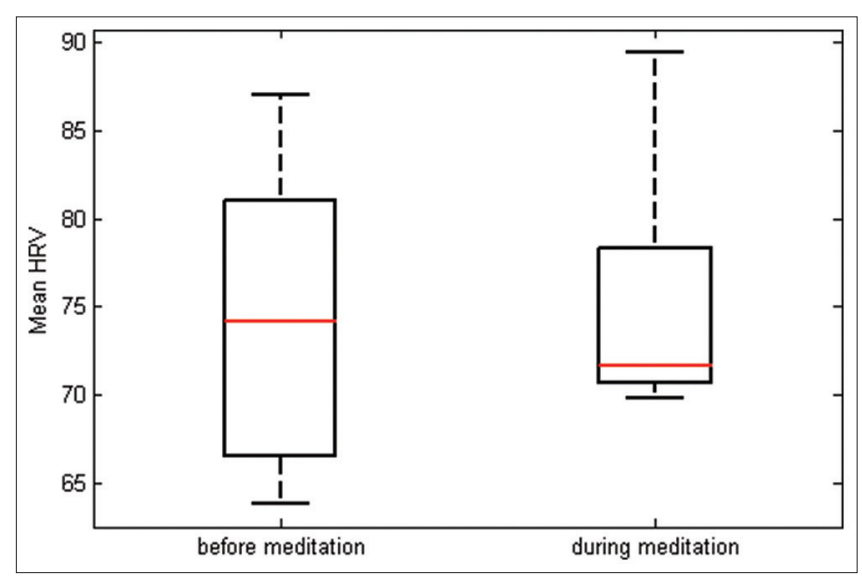

Figure 2: Mean HRV signals before and during meditation.

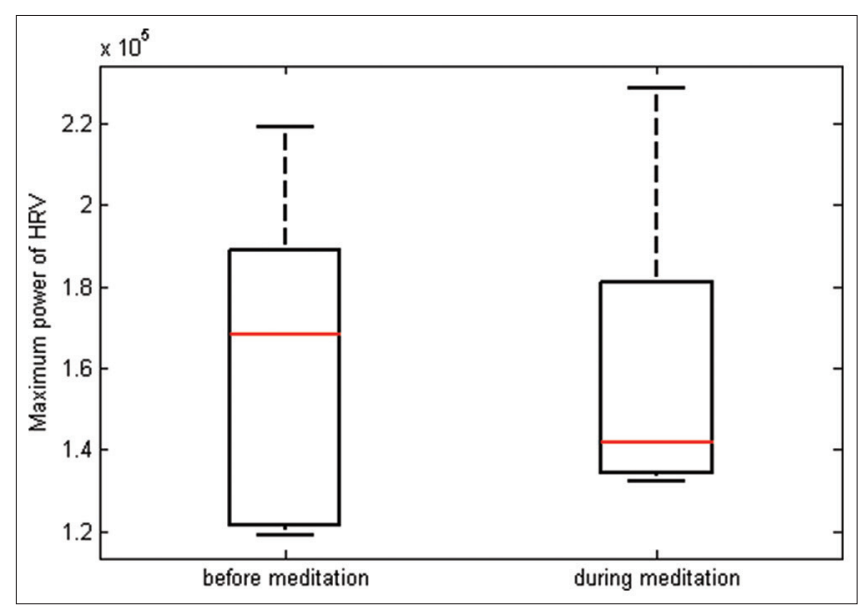

Figure 3: Maximum power spectrum of HRV before and during meditation. 
values, which tended to increase as the lag increased, the value of SD2 of Poincare plot did not change significantly. According to the results, the SD2 value with a lag of 1 was nearly $10.22 \pm 2.4$ and with a lag of 6 was about $10.02 \pm 2.5$. Therefore, as the lag increased, the shape of the plots became more circular during meditation.

The ratios of SD1/SD2 measured from Poincare plots reconstructed with lags of 1 and 6 , for all subjects, are shown in Figure 4A and B, respectively. As the graphs reveal, we can obviously notice that the values of SD1/SD2 ratio increased significantly during meditation compared to that before meditation, especially the index measured from Poincare plots reconstructed with a lag of $6(p<0.05)$.

Figure 5A and B shows the area of Poincare plot, reconstructed with lags of 1 and 6 , for both groups, i.e. before meditation and during meditation, respectively. The graphs demonstrate that in both lags, the area of Poincare plots decreased significantly during meditation compared to before meditation $(p<0.05)$. These decrements are more obvious in the lag of 1 heartbeat. Furthermore, the difference between the areas of Poincare plots tended to decrease as the lag increased.

\section{DISCUSSION}

In this study, we examined the influence of different lags on the Poincare plot indices, of HR signals, in the specific psychophysiological state. The SD1/SD2 and the area of the ellipse fitted to the Poincare plot were analyzed in a group of healthy subjects before and during meditation. These indices were estimated from Poincare plots reconstructed with lags of 1 and 6 heartbeats.

The SD1 index, which denotes the transverse axis of the Poincare plot, represents the standard deviation of the instantaneous variability of the beat-to-beat $\mathrm{HR}^{[10]}$ and provides supplementary information about the parasympathetic activity of cardiovascular system under the autonomic nervous system, ${ }^{[9,11]}$ whereas the SD2 values demonstrate the overall variability of RR intervals. ${ }^{[10,18]}$

Unlike SD1 values, which tended to increase as the lag increased, the value of SD2 of Poincare plot did not change significantly. According to the results, as the lag increased, the shape of the plots became more circular during meditation.

The results of our previous study ${ }^{[11]}$ demonstrate that the adjusted ellipses have different SD1 values before and during meditation. In healthy individuals before meditation, the beats' intervals are highly irregular, especially by the vagal predominance in heart modulation, which makes it possible to see a cloud of points with the approximate cigar shape in Poincare plot, and can be observed before meditation with a lag of 1 . Unlike before meditation, analysis of figures during meditation shows less spreading of RR intervals, both beat-to-beat and in the long term, and suggests lower HRV. In addition, as the lag increases, the shape of the plots becomes more circular during meditation. ${ }^{[1]}$ Furthermore, the center of the ellipse of

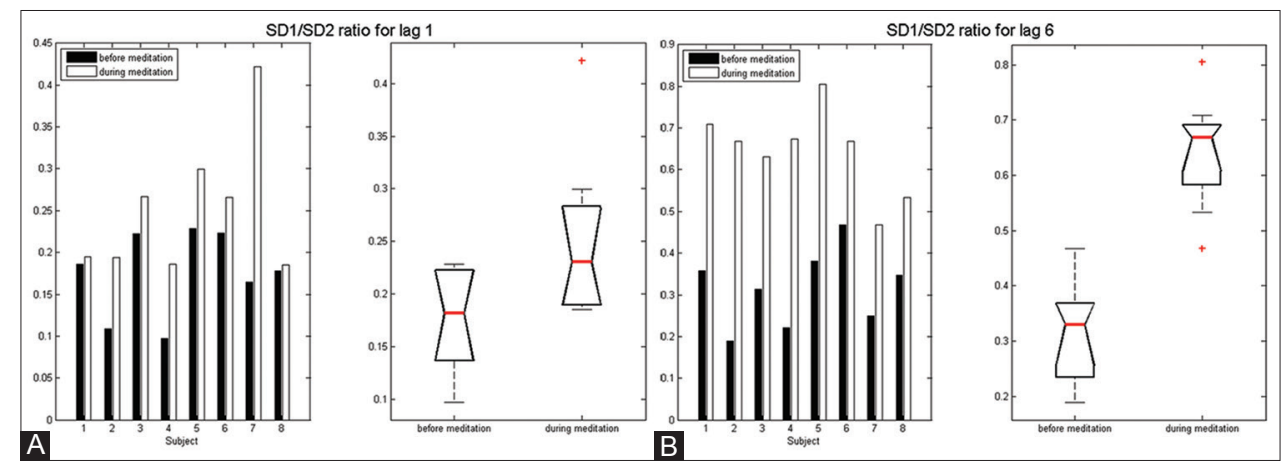

Figure 4: Poincare plot indices (SD1/SD2 ratio) of heart rate signals measured from the Poincare plots reconstructed with (A) Lag of 1 heartbeat and (B) lag of 6 heartbeats.

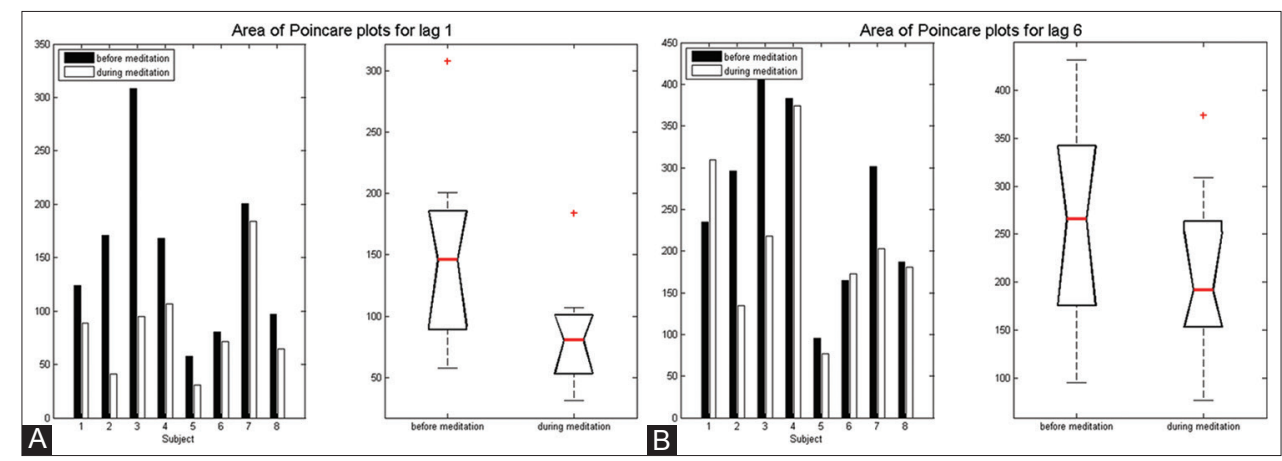

Figure 5: The area of Poincare plot of heart rate signals reconstructed with (A) Lag of 1 heartbeat and (B) lag of 6 heartbeats. 
Poincare plots shifts to lower values during meditation with respect to the center of the ellipse before meditation.

In a previous study, ${ }^{[11]}$ we focused on the minor axis of the ellipse and the influence of longer lags (up to six beats) over SD1. As the width (SD1) and length (SD2) of the Poincare plot express different information of the signal, in this study, based on both measures of SD1 and SD2, other indices of the Poincare plot (SD1/SD2 ratio and the area) were evaluated. Concerning the SD1/SD2 ratio measured from Poincare plots, the analyses show that the values of SD1/SD2 ratio increased significantly during meditation compared to that before meditation, especially the index measured from Poincare plots reconstructed with a lag of $6(p<0.05)$. Therefore, the SD1/SD2 ratio measured at a lag of 6 heartbeats could be useful to capture dynamic changes occurring during meditation.

The area of the ellipse which is fitted to the Poincare plot represents the total variability of HRV and it is under strong vagal influence. SD1/SD2 estimates the sympathetic-parasympathetic balance during meditation. ${ }^{[9,22]}$ The graphs show that in both lags, area of Poincare plots decreased significantly during meditation compared to before meditation $(p<0.05)$. Furthermore, the difference between the areas of Poincare plots tended to decrease as the lag increased.

Previously, Lerma et al. ${ }^{[23]}$ examined the Poincare plot indexes (SD1, SD2, and SD1/SD2) to discover dynamic fluctuations of the HRV after hemodialysis (HD) in hemodynamically stable chronic renal failure (CRF) patients. The results indicated that the Poincare plot indexes measured from CRF patients were smaller than those of healthy subjects. In addition, with a lag of 4 , the SD1/SD2 ratio of CRF patients reduced after HD. In another study, to extract temporal dynamics of Poincare plot, Karmakar et al. ${ }^{[19]}$ proposed a quantitative measure called Complex Correlation Measure (CCM). They compared this measure to SD1 and $\mathrm{SD} 2$ to evaluate the performance of the proposed index in differentiating arrhythmia and CHF patients from normal subjects. It was claimed that CCM can be applied as an additional Poincare plot descriptor to identify pathology. The correlations between SD1 and spectral HRV indices of type 1 diabetic patients were examined by Contreras et al..$^{[24]}$ It has been demonstrated that in the control group, SD1 with lags of 1 and 2 was extremely correlated with HF, whereas SD1 with lags of more than 4 was better correlated with LF rather than with HF. Though, in the diabetic group, the correlation between SD1 and spectral indices did not change as in the control group for different lags.

In conclusion, the comparative dynamic measures of the Poincare plot indices during and before meditation give more insight of the HR signals in a specific psychophysiological state. The difference between these features during meditation in comparison with before meditation might be useful for analysis of HR signals during meditation.
There may be some limitations that need to be considered in the future studies. For instance, even though the database has been frequently used in the recent scientific researches, ${ }^{[11,15,25]}$ more data should be examined to generalize the findings. Further investigations can employ more varied features and other meditation techniques.

\section{REFERENCES}

1. Peng CK, Mietus JE, Liu Y, Khalsa G, Douglas PS, Benson H, et al. Exaggerated heart rate oscillations during two meditation techniques. Int J Cardiol 1999;70:101-7.

2. Nolan J, Batin PD, Andrews R, Lindsay SJ, Brooksby P, Mullen M, et al. Prospective study of heart rate variability and mortality in chronic heart failure: Results of the United Kingdom heart failure evaluation and assessment of risk trial (UK-heart). Circulation 1998;98:1510-6.

3. Lanza GA, Pedrotti P, Rebuzzi AG, Pasceri V, Quaranta G, Maseri A. Usefulness of the addition of heart rate variability to Holter monitoring in predicting in-hospital cardiac events in patients with unstable angina pectoris. Am J Cardiol 1997;80:263-7.

4. Kleiger RE, Miller JP, Bigger JT Jr, Moss AJ. Decreased heart rate variability and its association with increased mortality after acute myocardial infarction. Am J Cardiol 1987;59:256-62.

5. Rajendra Acharya U, Paul Joseph K, Kannathal N, Lim CM, Suri JS. Heart rate variability: A review. Med Biol Eng Comput 2006;44:1031-51.

6. Raghavendra BS, Dutt DN. Nonlinear dynamical characterization of heart rate variability time series of meditation. World Academy of Science, Engineering and Technology 2011;5:952-63.

7. Khaled AS, Owis MI, Mohamed AS. Employing time-domain methods and Poincaré plot of heart rate variability signals to detect congestive heart failure. BIME Journal 2006;6:35-41.

8. Voss A, Schroeder R, Truebner S, Goernig M, Figulla HR, Schirdewan A. Comparison of nonlinear methods symbolic dynamics, detrended fluctuation, and Poincaré plot analysis in risk stratification in patients with dilated cardiomyopathy. Chaos 2007;17:015120.

9. Tulppo MP, Makikallio TH, Takala TE, Seppanen T, Huikuri HV. Quantitative beat-to-beat analysis of heart rate dynamics during exercise. Am J Physiol 1996;271:H244-52.

10. Brunetto AF, Silva BM, Roseguini BT, Hirai DM, Guedes DP. Limiar ventilatório e variabilidade da frequência cardíaca em adolescentes. Rev Bras Med Esporte 2005;11:22-7.

11. Goshvarpour A, Goshvarpour A, Rahati S. Analysis of lagged Poincare plots in heart rate signals during meditation. Digit Signal Process 2011;21:208-14.

12. Jevning R, Wallace RK, Beidebach M. The physiology of meditation - A review - A wakeful hypometabolic integrated response. Neurosci Biobehav Rev 1992;16:415-24.

13. Sun TF, Wu CK, Chiu NM. Mindfulness meditation training combined with eye movement desensitization and reprocessing in psychotherapy of an elderly patient. Chang Gung Med J 2004;27:464-9.

14. Phongsuphap S, Pongsupap Y, Chandanamattha P, Lursinsap C. Changes in heart rate variability during concentration meditation. Int J Cardiol 2008;130:481-4. 
15. Goshvarpour A, Goshvarpour A. Comparison of higher order spectra in heart rate signals during two techniques of meditation: Chi and Kundalini meditation. Cogn Neurodyn 2013;7:39-46.

16. Muralikrishnan K, Balakrishnan B, Balasubramanian K, Visnegarawla F. Measurement of the effect of Isha Yoga on cardiac autonomic nervous system using short-term heart rate variability. J Ayurveda Integr Med 2012;3:91-6.

17. Litscher G, Zhang W, Huang T, Wang L. Heart rate and heart rate variability responses to Tai Chi and jogging in Beijing and Graz. N Am J Med Sci 2011;3:70-4.

18. Task Force of the European Society of Cardiology and the North American Society of Pacing and Electrophysiology: Heart rate variability: Standards of measurement, physiological interpretation and clinical use. Circulation 1996;93:1043-65.

19. Karmakar CK, Khandoker AH, Gubbi J, Palaniswami M. Complex correlation measure: A novel descriptor for Poincare plot. Biomed Eng Online 2009;8:17.

20. Brennan M, Palaniswami M, Kamen P. Do existing measures of Poincare plot geometry reflect nonlinear features of heart rate variability? IEEE Trans Biomed Eng 2001;48:1342-7.

21. Vanderlei LC, Pastre CM, Hoshi RA, Carvalho TD, Godoy MF. Noções básicas de variabilidade da frequência cardíaca e sua aplicabilidade clínica. Rev Bras Cir Cardiovasc 2009;24:205-17.

22. Tulppo MP, Makikallio TH, Seppanen T, Laukkanen RT, Huikuri HV. Vagal modulation of heart rate during exercise: Effects of age and physical fitness. Am J Physiol 1998;274:H424-9.

23. Lerma C, Infante O, Perez-Grovas H, Jose MV. Poincare plot indexes of heart rate variability capture dynamic adaptations after haemodialysis in chronic renal failure patients. Clin Physiol Funct Imaging 2003;23:72-80.

24. Contreras P, Canetti R, Migliaro ER. Correlations between frequency-domain HRV indices and lagged Poincare plot width in healthy and diabetic subjects. Physiol Meas 2007;28:85-94.

25. Song R, Bian C, Ma QD. Multifractal analysis of heartbeat dynamics during meditation training. Physica A: Statistical Mechanics and its Applications 2013;392:1858-62. 